\title{
Primary enteric adenocarcinoma with predominantly signet ring features of the lung: A case report with clinicopathological and molecular findings
}

\author{
Makoto Nagashima ${ }^{1}$, Ayako Moriyama ${ }^{1}$, Yasuo Matsuzawa ${ }^{2}$, Noriaki Okada ${ }^{2}$, Kensuke Terai ${ }^{3}$, \\ Daisuke Sasai $^{3}$, Wataru Tokuyama ${ }^{3}$, Nobuyuki Hiruta ${ }^{3}$, Ryoji Katoh ${ }^{1}$ \\ 1. Department of Surgery, Toho University Sakura Medical Center, Sakura, Japan. 2. Department of Internal Medicine, \\ Respiratory \& Allergy Division, Toho University Sakura Medical Center, Sakura, Japan. 3. Department of Pathology, Toho \\ University Sakura Medical Center, Sakura, Japan.
}

Correspondence: Makoto Nagashima. Address: Department of Surgery, Toho University Sakura Medical Center, 564-1 Shimoshizu, Sakura, Japan. E-mail: nagashima@sakura.med.toho-u.ac.jp

Received: February 26, 2015

DOI : $10.5430 /$ crcp.v2n3p90
Accepted: May 8, 2015

URL: http://dx.doi.org/10.5430/crcp.v2n3p90

\section{Abstract}

Primary signet ring adenocarcinoma (SRA) of the lung is a rare condition. An 81-year-old man was referred to our hospital because of bloody sputum. Chest radiography showed a tumor in the right lung and biopsies revealed typical SRA. No tumors except for the one in the right lung were found. Therefore, diagnosis of primary lung SRA was made. The SRA cells showed intracytoplasmic mucin that were characterized by crescentic nuclei displaced toward one end of the cell. The tumor cells showed negative immunostaining for thyroid transcription factor 1 (TTF-1), but positive immunostaining for CDX2, cytokeratin-20 (CK20), Ki-67 (labeling = 50\%), p53, and focally CK7. A right lower lobectomy and lymph node dissection were performed via video-assisted thoracic surgery. After surgery, uracil-tegafur $\left(184 \mathrm{mg} / \mathrm{m}^{2} / \mathrm{day}\right)$ was administered for five days, followed by a 2-day rest period for a duration of two years in total. No recurrence was observed during the monthly-follow ups, and the patient did not experience any complications. In conclusion, we describe an extremely rare case of primary enteric adenocarcinoma with predominantly signet ring features of the lung. Our case report includes in-depth immunohistochemical and molecular data.

\section{Key words}

Signet ring adenocarcinoma, Lung cancer

\section{I ntroduction}

Signet ring adenocarcinoma (SRA), an abundant intracellular mucin-producing adenocarcinoma, originates in various organs, including the stomach, colon, urinary bladder, prostate, and breast. However, primary pulmonary SRA is a rare condition. SRA is characterized by crescentic nuclei that are displaced toward one end of the cells ${ }^{[1-5]}$. Extensive immunohistochemistry (IHC) and molecular studies of primary SRA of the lung have not been performed yet. We herein report a case of an extremely rare case of primary enteric adenocarcinoma with predominantly signet ring features of the lung and provide an in-depth description of the clinicopathological and molecular characteristics. 


\section{Case report}

An 81-year-old man was referred to our hospital because of bloody sputum. Chest radiography showed a tumor in the right lung. Levels of tumor markers (CEA, CA19-9, DUPAN-2, SLX, Span-1) were within the normal range. Computed tomography (CT) demonstrated a tumor with a size of $40 \mathrm{~mm}$ in the right lower lobe of the lung (see Figure 1). 18F-fluorodeoxyglucose-positron emission tomography (FDG-PET) showed activity in this lesion with a maximum standardized uptake value of 2.4. A transbronchial endoscopy revealed a tumor in the right lower bronchus. Lung biopsies of the bronchial lesions revealed typical SRA. Upper and lower gastrointestinal endoscopies revealed no tumors and a head CT demonstrated no brain metastases. In summary, the imaging modalities did not demonstrate other tumors than the one in the right lung. Therefore, the tumor in the patient's right lung was diagnosed as a primary lung SRA. The clinical TNM classification was T2N0M0, cStage IB.

Figure 1. Computed tomography demonstrated a nodule, measuring $40 \mathrm{~mm}$ in diameter in the right lower lobe (density in the lung)

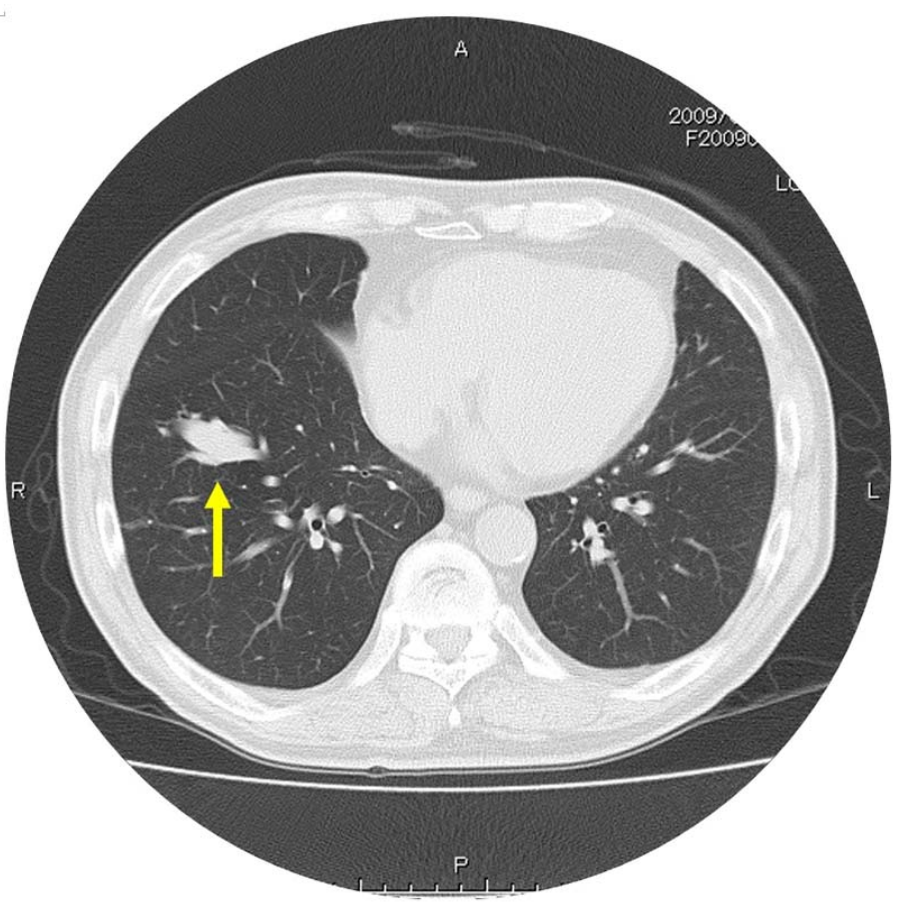

Macroscopically, the tumor ( $50 \mathrm{~mm} \times 35 \mathrm{~mm} \times 18 \mathrm{~mm}$ in size) was located in the right lower lobe of the lung and had a distinct boundary. Histopathologically, the tumor cells revealed typical SRA cells showing intracytoplasmic mucin and characterized by the presence of crescentic nuclei that were displaced toward one end of the cells (see Figure 2). Partially, the tumor consisted of papillotubular adenocarcinoma, and showed a transition to signet ring features. Genetic analysis revealed the presence of a wild-type EGFR gene in the tumor cells. No ALK protein expression was observed by IHC using a rabbit monoclonal antibody to ALK and that fluorescence in situ hybridization (FISH) analysis confirmed the absence of ALK rearrangement. Hence, the SRA in this patient was not an EML4-ALK positive lung adenocarcinoma. Immunohistochemically, the tumor cells were positive for CDX2, CK20, Ki-67 (labeling = 50\%), p53, and focally CK7, but negative for TTF-1, mucin-6 (MUC6) and HIK1083 (see Figure 3). A pathological diagnosis of a primary enteric adenocarcinoma with predominantly signet ring features of the right lung was made.

A right-lower lobectomy and lymph node dissection were performed via video-assisted thoracic surgery. This surgery was curative. Postsurgery, uracil-tegafur was administered orally at a dose of $184 \mathrm{mg} / \mathrm{m}^{2} /$ day for five days, followed by a 2-day rest period for a duration of two years in total.

No residual disease was detected on a CT scan after the patient's surgery. During his monthly follow-ups, no recurrences in any organs were noted. The patient is now 86 years old and is living a healthy life without any complications. 

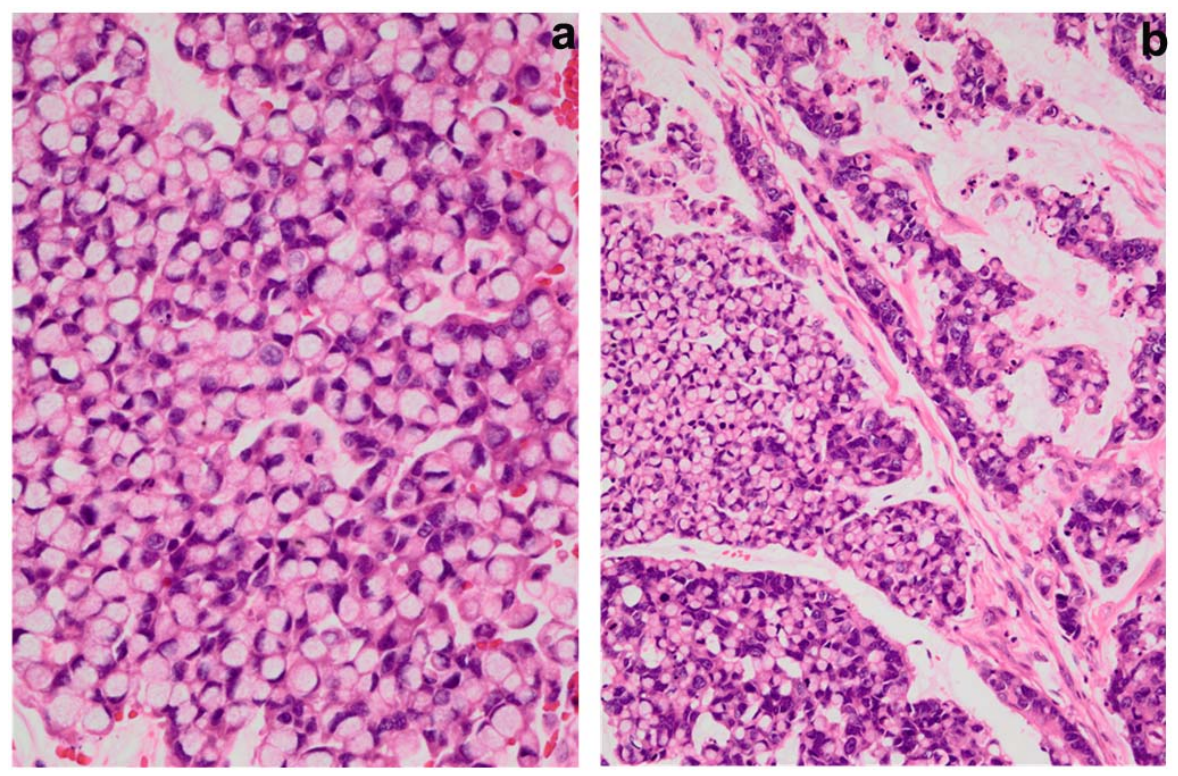

Figure 2. Histological findings. a. The almost of tumor is the feature of so-called signet ring adenocarcinoma. The cells show intracytoplasmic mucin and crescentic nuclei that displace toward one end of the cells. b. Partially, the tumor consists of papillotubular adenocarcinoma, and shows a transition to signet ring features.
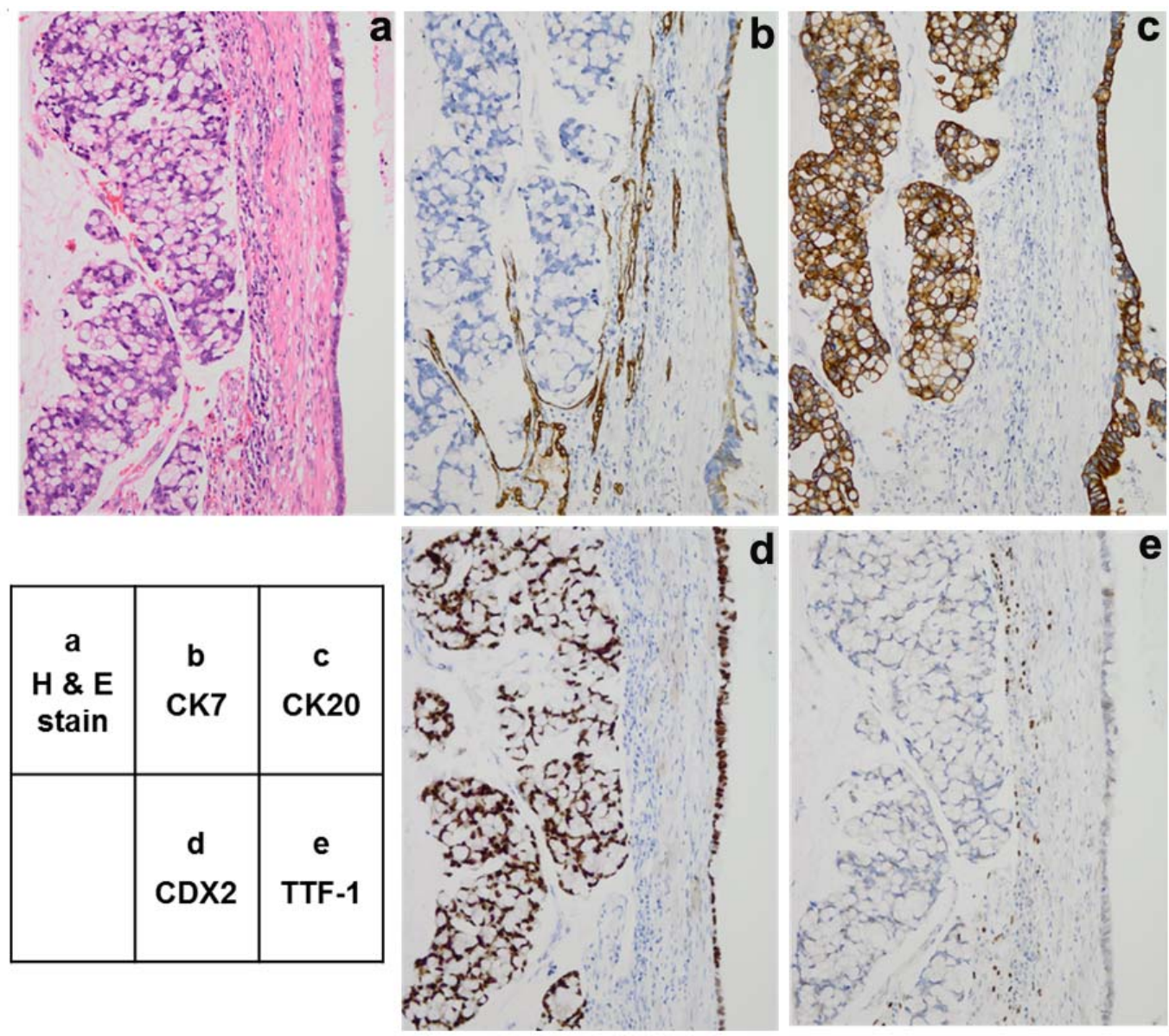

Figure 3. Immunohistochemical findings. a. In Hematoxylin \& Eosin stain, the signet ring features are found in the left side, and the atypical columnar epithelium is found in the right side. b. CK7 is focally positive in the columnar epithelium, but is negative in a signet ring area. c. CK20 shows positive. d. CDX2 shows positive. e. TTF-1 is negative. 


\section{Discussion}

In most cases of gastric and lung SRA, SRA cells are present in addition to other histological subtypes such as tubular adenocarcinoma and mucinous adenocarcinoma. In general, an adenocarcinoma with more than $50 \%$ signet ring cell carcinoma cells is called SRA ${ }^{[3]}$. It is important to exclude metastatic SRA that has originated in the stomach, breast and other organs in the diagnosis of extragastric SRA. In the present case, all organs besides the lung were free from tumors, as shown by radiology. Thus, the patient was diagnosed with primary SRA of the lung.

The clinicopathological features of pulmonary SRA have not been sufficiently clarified, as this condition is so rare. According to the study with the largest sample size $(n=39)$, lung carcinoma with signet ring cell components accounted for $1.5 \%(39 / 2,640)$ of all lung malignancies ${ }^{[3]}$. The patients' mean age was 54.6 years (range 32-76 years), the male to female ratio was 1.16:1.00, and the 5-year survival was $28 \%$. The prognosis of lung SRA is poor. Several IHC studies of lung SRA have been published ${ }^{[2,4,5]}$. Merchant et al. indicated that $82.4 \%$ of patients (14/17) showed positive TTF-1 expression, and a CK7(+)/CK20(-) expression pattern was detected in $94 \%$ of patients (16/17) ${ }^{[2]}$. Castro et al. showed TTF-1 (100\%, 6/6), CEA (100\%, 9/9), and CK7 (50\%, 3/6) immunoreactivity, whereas no CK20 expression was observed ${ }^{[1]}$. In the present case, we detected negative TTF-1 expression by using IHC. Overall, we observed the following immunoreactivity pattern: CDX2(+), CK7(+, focally), CK20(+), MUC6(-) and HIK1083(-). This immunoreactivity pattern is not consistent with primary lung adenocarcinoma, but is consistent with primary intestinal adenocarcinoma. Thus, pathological and immunohistochemical diagnosis of primary enteric adenocarcinoma with predominantly signet ring features of the lung was made. In the present case, the Ki-67 labeling index was high (50\%) and p53 immunoreactivity was positive, indicating active cell proliferation and the presence of p53 mutations, respectively.

Recently, the EML4-ALK fusion gene was detected in non-small cell lung cancer. The EML4-ALK fusion gene encodes a constitutively active oncoprotein in cancer cells. Some studies have indicated that EML4-ALK positive lung adenocarcinomas, in particular the acinar and signet ring cell subtype, typically have a histologically less-differentiated grade ${ }^{[6,7]}$. In this study, we did not detect ALK protein on IHC or ALK rearrangement on FISH analysis. Thus, the SRA in our case was not an EML4-ALK-positive lung adenocarcinoma.

ROS1 rearrangements have been identified in glioblastomas and non-small cell lung cancer. ROS1 proto-oncogene translocation leads to a constitutive alteration of the kinase and activates downstream signaling of several oncogenic pathways ${ }^{[8]}$. In the present case, no ROS1 expression was detected on IHC. Thus, the tumor was not a ROS1-rearranged lung adenocarcinoma. In summary, the driver mutations of EGFR, ALK, and ROS1 were not detected in this primary pure lung SRCA.

In conclusion, we describe an extremely rare case of primary enteric adenocarcinoma with predominantly signet ring features of the lung, along with in-depth an immunohistochemical and molecular data.

\section{Acknowledgments}

This work was supported by Grants-in-Aid from the Smoking Research Foundation.

\section{Conflict of interests}

The authors have no financial conflict of interests.

\section{References}

[1] Castro CY, Moran CA, Flieder DG, et al. Primary signet ring cell adenocarcinomas of the lung: a clinicopathological study of 15 cases. Histopathology. 2001; 39(4): 397-401. PMid: 11683941. http://dx.doi.org/10.1046/j.1365-2559.2001.01224.x 
[2] Merchant SH, Amin MB, Tamboli P, et al. Primary signet-ring cell carcinoma of lung: immunohistochemical study and comparison with non-pulmonary signet-ring cell carcinoma. Am J Surg Pathol. 2001; 25(12): 1515-1519. PMid: 11717541. http://dx.doi.org/10.1097/00000478-200112000-00007

[3] Tsuta K, Ishii G, Yoh K, et al. Primary lung carcinoma with signet-ring cell carcinoma components: clinicopathological analysis of 39 cases. Am J Surg Pathol. 2004; 28(7): 868-874. PMid: 15223955. http://dx.doi.org/10.1097/00000478-200407000-00004

[4] Tsuta K, Ishii G, Nitadori J, et al. Comparison of the immunophenotypes of signet-ring cell carcinoma, solid adenocarcinoma with mucin production, and mucinous bronchioloalveolar carcinoma of the lung characterized by the presence of cytoplasmic mucin. $\mathrm{J}$ Pathol. 2006; 209(1): 78-79. PMid: 16463270. http://dx.doi.org/10.1002/path.1947

[5] Terada T. Primary pure signet-ring cell carcinoma of the extrapulmonary left main bronchus: a case report with an immunohistochemical study. Int J Clin Exp Pathol. 2013; 6(6): 1138-1141. PMid: 23696933.

[6] Ohba T, Sugio K, Kometani T, et al. Signet ring cell adenocarcinoma of the lung with an EML4-ALK fusion gene mimicking mucinous (colloid) adenocarcinoma: a case report. Lung Cancer. 2011; 73(3): 375-378. PMid: 21719143. http://dx.doi.org/10.1016/j.lungcan.2011.05.016

[7] Pareja F, Crapanzano JP, Mansukhani MM, et al. Cytomorphological features of ALK-positive lung adenocarcinoma: psammoma bodies and signet ring cells. Cancer Cytopathol. 2014. http://dx.doi.org/10.1002/cncy.21507

[8] Pan Y, Zhang Y, Li Y, et al. ALK, ROS1 and RET fusions in 1139 lung adenocarcinomas: a comprehensive study of common and fusion pattern-specific clinicopathologic, histologic and cytologic features. Lung Cancer. 2014; 84(2): 121-126. PMid: 24629636. http://dx.doi.org/10.1016/j.lungcan.2014.02.007 